\title{
Распространение потока носителей заряда в тонком слое плоскопараллельной твердотельной структуры с учетом рассеяния на границах слоя
}

\author{
(C) М.Б. Керими
}

Центр технологий Академии наук Туркменистана, 744000 Ашхабад, Туркменистан

E-mail: mb8krmi@gmail.com

(Получена 30 ноября 2016 г. Принята к печати 25 января 2017 г.)

Строение границ тонкого слоя плоскопараллельной твердотельной структуры влияет на распространение дифференциальных потоков носителей заряда в толще слоя и сквозь него. Рассеяние этих потоков на границах тонкого слоя влияет и на функцию распределения носителей заряда. Это рассеяние корректно учтено в интегральных граничных условиях для дифференциальных потоков к кинетическому уравнению. Для плоскопараллельного слоя кинетическое уравнение в приближении времени релаксации сведено к удобной форме, описывающей распространение дифференциальных потоков в толще слоя. В общем виде получено решение задачи о распространении дифференциальных потоков носителей заряда в слое структуры. Проведен анализ решения для толстых и тонких слоев разного типа - металла, диэлектрика, полупроводника. Определены востребованные практикой задачи, в которых можно продуктивно использовать полученные решения.

DOI: 10.21883/FTP.2017.08.44797.8156

\section{1. Введение}

Многие современные твердотельные структуры, в том числе структуры солнечных элементов и фотодетекторов, имеют тонкие плоскопараллельные слои субмикронных и наноразмеров. Обычно такие структуры, в частности структуры каскадного солнечного элемента, создаются наращиванием каждого последующего слоя на предыдущем. Предыдущий слой является подложкой для последующего. При этом слой-,подложка“ не всегда оказывается идеальным для наращивания следующего слоя структуры с требуемыми электрическими, фотоэлектрическими или оптическими свойствами. Поверхность каждого выращенного тонкого слоя может получиться со сложным строением, в частности с гомогенным или негомогенным микрорельефом. На границах могут появиться распределения некоторых наномасштабных неоднородностей, в том числе мелких пор, дефектов, кластеров и т.п. В частности, строение поверхности тонкого токопроводящего слоя $\mathrm{ZnO}$ на солнечном элементе [1] существенно зависит от технологии его получения и примесей легирования. Наличие неконтролируемых или специально созданных особенностей в строении границ влияет на распространение потоков носителей заряда у границы и через нее, а также на потоки в тонком слое и структуре в целом. Поэтому исследование распространения потоков носителей в тонких слоях представляет большой научный и практический интерес.

Как показывают многочисленные исследования поверхностей и границ твердотельных слоев [1-10], в наномасштабе они обычно имеют сложное строение. В частности, границы могут иметь специально созданные или слабо технологически контролируемые мик- рорельеф, распределение различных фаз, пор и других мелких неоднородностей или нанообъектов, которые влияют на пространственное и энергетическое строение границы или поверхности.

Наличие различных пространственных размеров у элементов микрорельефа и его особенностей на границе - глубины проникновения в толщу слоя и поперечных размеров его составляющих (собственного вещества, возможных фазовых образований, разных типов дефектов, инородных нанообъектов, приграничных электронных состояний, неоднородностей полей и т. п.), a также гомогенный или негомогенный характер их распределения в масштабах рассматриваемой теории могут влиять на корректность допущения, что рассматриваемый слой является тонким и плоскопараллельным.

Очевидно, слой можно считать тонким в рамках используемой теории, если его толщина $d$ сопоставима с характеристической длиной $l$ этой теории. Слой корректно считать тонким и плоскопараллельным в рамках данной теории, если из-за наличия микрорельефа на границах дисперсия толщины слоя локально и по всей его площади очень мала, так, что среднеквадратичное отклонение толщины много меньше длины $l$ и $d$, поперечные размеры всех видов неоднородностей тоже много меньше $l$, и локальные свойства границы формируются их гомогенным распределением, а также если свойства обеих границ вдоль слоя, как и вдоль элементарных сечений толщи слоя $(d x \ll l)$, практически не меняются. Очевидно, что способность рассеивать дифференциальные потоки носителей зарядов границей плоскопараллельного слоя, характеризуемая соответствующей вероятностью, должна быть однородной по границе. Кроме этого, угловая азимутальная зависимость вероятности 
рассеяния потоков должна быть еще и равновероятной, максимально симметричной по отношению к нормали макрограницы. В противном случае „плоскопараллельность“ слоя на границе нарушается.

\section{2. Способы описания распространения потоков носителей в тонком слое}

Теоретическое описание распространения потоков носителей заряда в тонком слое должно учитывать преобладающие процессы, происходящие с носителями в слое и на его границах, а также соответствовать требуемой детализации описания. Описание может быть квантово-механическим (сложным), микроскопическим (менее сложным), или феноменологическим (относительно простым). Хотя первое из названных описаний применимо во всех случаях и разных толщинах, из-за больших сложностей его используют редко при расчетах в „толстом“ слое. Применение остальных описаний имеет ограничения, в том числе по минимальной толщине слоя. Условие однородности свойств на границе и в элементарных слоях толщи в латеральном направлении („плоскопараллельность“ слоя) наиболее просто удовлетворяется современными массовыми технологиями в рамках феноменологической, менее детальной теории, поскольку диффузионно-дрейфовая длина $L_{e}$ намного больше длины свободного пробега $l_{e}$ и квантовомеханической длины волны носителей $\lambda_{e}$. В рамках квантовой механики удовлетворить условие гомогенности распределения нанонеоднородностей на границах и в толще слоя на расстояниях порядка $\lambda_{e}$ удается не всегда, как в силу необходимости создания требуемых свойств, например легирования, так и из-за особенностей, характерных для современного развития технологий создания слоев на основе конкретных твердотельных материалов и на подложках. Кинетическая теория в этом отношении занимает промежуточное положение, поскольку обычно $\lambda_{e}<l_{e} \ll L_{e}$.

В тонких слоях возможно баллистическое или квазибаллистическое распространение носителей заряда. В этом случае рассеяние потока носителей происходит в основном на границах тонкого слоя. Поэтому роль рассеяния потоков на границах значительно возрастает. При этом диффузия и дрейф носителей в тонком слое имеют особенности. В латеральном направлении и поперек слоя они отличаются, поскольку характер рассеяния дифференциальных потоков носителей на границах, зависящий от их пространственного и энергетического строения и состояния, в общем случае неодинаков с „объемным“. Следовательно, при феноменологическом описании распространения потоков в тонких слоях порядка 2-3 длины свободного пробега $l_{e}$ нельзя использовать обычные „объемные“ значения коэффициентов диффузии и подвижности. Если угадать некие эффективные значения указанных коэффициентов, которые зависят от строения и состояния границ слоя, а также от его толщины, то, возможно, рассматриваемое описание будет корректным.

Если же допущение „слой является тонким и плоскопараллельным в рамках микроскопической теории“ как локально, так и по всей площади слоя статистически хорошо выполняется, то в рассматриваемом слое возможно корректное и достаточно простое описание распространения потоков в рамках кинетической теории с учетом рассеяния потоков на границах слоя [11-13]. В указанных работах напряженность электрического поля в слое, возникающая из-за приложенной к слою „внешней“ разности потенциалов, предполагалась постоянной. В настоящей работе рассматривается более общий случай изменения напряженности электрического поля в слое.

В сверхтонких слоях, поперечные размеры которых сопоставимы с несколькими постоянными кристаллической решетки, корректным является квантовомеханическое описание. При вычислении вероятностей рассеяния носителей заряда границей также необходимо указанное описание. В этом случае вопрос о наличии локальной и/или макроскопической „плоскопараллельности“, т.е. о характере изменения свойств и форме границы, а также о корректном задании граничных условий необходимо решать с учетом конкретного пространственного и энергетического строения и состояния слоя и его границ [14]. Наличие локальных сверхтонких участков в тонком слое, имеющем статистически плохо обусловленную толщину порядка $l_{e}$, может привести к появлению заметных туннельных токов наряду с обычным током.

В силу перечисленного кинетическая теория с корректными граничными условиями в тонком слое сочетает в себе достаточную детальность и точность описания, а также имеет менее строгие, чем квантовомеханические, ограничения размеров области усреднения характеристик свойств толщи и границы (гомогенности). Поэтому далее основное внимание будет уделено именно кинетическому описанию распространения потоков носителей заряда в тонких плоскопараллельных слоях.

Наличие распределений неоднородностей на границе, в том числе, например, специально внедренных нанокластеров, влияет на пространственное и энергетическое строение зон, а также на строение граничного потенциала. В частности, даже строение „естественного“ микрорельефа может влиять на расщепление энергетических зон на границе и неоднородную их зависимость вдоль границы. Это связано с характером локального нарушения ближнего порядка по границе, который, как известно, в основном и определяет строение энергетических зон в атомарно ближней окрестности. Зависимость зоны проводимости $E_{C}(\mathbf{r})$, валентной зоны $E_{V}(\mathbf{r})$, „Поверхностных“ граничных уровней $E_{S}(\mathbf{r})$ и локальной координатной зависимости ширины запрещенной зоны $E_{g}(\mathbf{r})$ по границе, как и электрического потенциала [5], при наличии наномасштабной гомогенности 
микрорельефа в общем случае можно считать статистически стационарными случайными функциями, если отклонения указанных функций от средних значений невелики.

Данные особенности строения зон, граничного потенциала, микрорельефа и распределения мелких неоднородностей могут существенно влиять на потоки носителей заряда у границы и через границу, и тем самым на функцию распределения носителей заряда вблизи границы. Если распределение различных мелких неоднородностей не проникает глубоко в слой, то их влияние необходимо корректно учитывать в граничных условиях к кинетическому уравнению через вероятности рассеяния для дифференциальных потоков носителей заряда. Корректное решение обсуждаемой проблемы в общем случае представляется весьма сложным изза наличия многих признаков в строении и состоянии границы, а также ее особой статистики [15].

\section{3. Интегральные граничные условия к кинетическому уравнению}

Рассмотрим некоторый внутренний твердотельный слой плоскопараллельной структуры. Пусть характерные размеры микрорельефа и размеры неоднородных областей $h$, включая глубину проникновения неоднородностей электрического поля, по границам слоя много меньше длины свободного пробега носителей заряда $l_{e}$. Пусть также разные типы неоднородностей достаточно плотно и равномерно распределены вдоль границы. В этом случае толщина рассматриваемого слоя $d$ является статистически хорошо обусловленной величиной, когда $d \geq l_{e}$, и свойства поверхности (или границы) являются гомогенизированными в рамках кинетической теории. Сложное состояние границы, включая строение микрорельефа, в этом случае можно корректно учесть в свойствах плоской макрограницы через рассеяние дифференциальных потоков носителей заряда на ней. При этом трехмерная в обычном пространстве задача сводится к плоскопараллельной, что существенно облегчает ее решение и интерпретацию результатов. В других случаях корректное описание распространения потоков носителей заряда через границу усложняется.

В равновесии известная функция Ферми-Дирака, характеризующая распределение электронов по их энергии в слое, является решением кинетического уравнения $[16,17]$. У границы физические условия равновесия отличаются от условий в объеме слоя, и преобладающие механизмы рассеяния у границы могут существенно отличаться от „объемных“. Поэтому в данной области необходимо учитывать влияние границы явно. Однако известные граничные „условия Фукса“ [17] для функции распределения в тонком плоскопараллельном слое учитывают влияние границы несколько ограниченно и не во всех случаях адекватно. Интегральные граничные условия для рассеивающихся на границе дифференциальных потоков носителей заряда, используемые ниже, интерпретируются физически проще и точнее [14].

Резкое локальное изменение энергетических зон на границе, реальный граничный потенциал со сложным слабонеоднородным профилем, наряду с микрорельефом и другими неоднородностями, соответствующими строению и состоянию границы в субмикронном и наномасштабе, через рассеяние носителей на границе влияют на электрохимический потенциал, и тем самым на функцию распределения электронов. Поскольку дифференциальные потоки носителей заряда зависят от их функции распределения, содержащей электрохимический потенциал, это влияние оказывается взаимным.

В рассматриваемом случае в силу небольшой глубины границы и ее гомогенности указанную взаимосвязь можно определить, если задать вероятность специфичного граничного рассеивания дифференциальных потоков, от которой зависят доли отраженного, выходящего и „потерянного“ потоков носителей заряда на границе. Вероятности рассеяния потоков должны соответствовать сложному состоянию границы со специфичным пространственным и энергетическим строением, статистике границы и сложившимся у границы физическим условиям, как при наличии тока, так и при его отсутствии.

Проекции дифференциальных потоков, падающих на „Плоскую“ границу слоя с разных ее сторон, имеют только позитивные или только негативные значения на нормаль границы. В толще обоих смежных слоев носители имеют волновой вектор всевозможных, в том числе противоположных, направлений. Поэтому любой результирующий дифференциальный поток в каждом слое вдоль волнового вектора $\mathbf{k}$ можно представить в виде разности двух потоков, направленных в противоположные стороны: $\mathbf{q}^{+}(\mathbf{r}, \mathbf{k})$ и $\mathbf{q}^{-}(\mathbf{r},-\mathbf{k})$. При этом на границу с одной стороны падают потоки $\mathbf{q}^{+}(\mathbf{r}, \mathbf{k})$, a c другой $-\mathbf{q}^{-}(\mathbf{r},-\mathbf{k})$ (далее опустим знак „минус“ в скобках, что не приведет к осложнениям).

Рассмотрим общую стационарную физическую картину, происходящую на границе раздела твердотельных слоев. На элементарную площадку $d \sigma$, выбранную в виде круга радиусом $h \ll r \ll l_{e}$ и содержащую достаточно много типичных для границы неоднородностей (размерами не более $h$ ), с разных направлений из толщи слоя падают потоки носителей заряда. Это происходит с носителями заряда в силу наличия у них квазиимпульса. Каждый падающий дифференциальный поток с некоторой вероятностью может дать вклад в некий фиксированный дифференциальный поток, появившийся в результате рассеяния. Из общих соображений ясно, что некоторая часть $R$ всех падающих на эту площадку потоков носителей отражается, часть $T$ проходит ее, и часть $S$ теряется на ней, например, из-за наличия рекомбинации. При этом, очевидно, должно выполняться условие $R+T+S=1$ для интегрального потока, падающего на площадку $d \sigma$ с каждой стороны. 


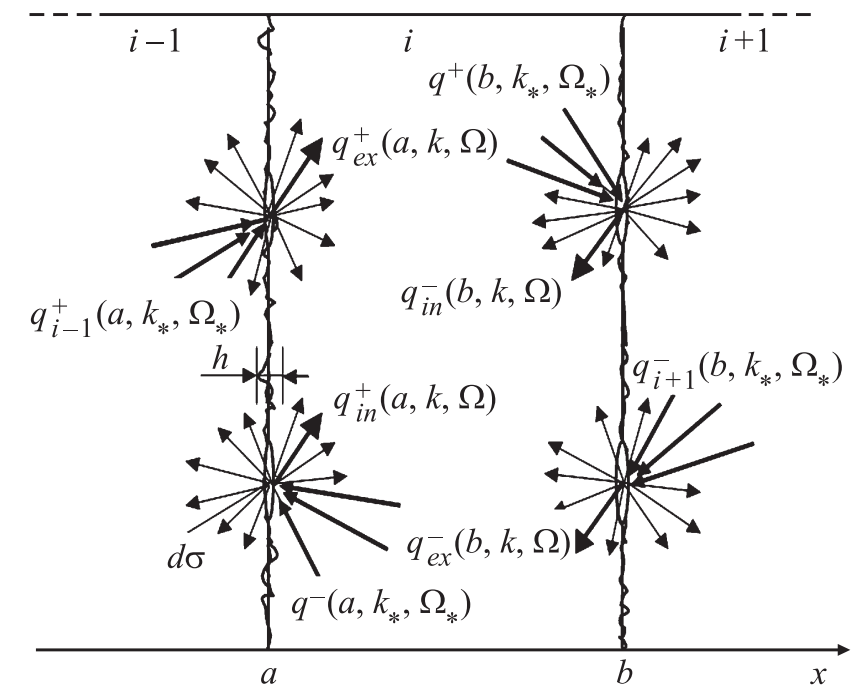

Схема слоев плоскопараллельной структуры и распространения дифференциальных потоков носителей заряда на границах некоторого внутреннего слоя.

Если рассматриваемый плоскопараллельный слой расположен внутри многослойной твердотельной структуры, то наряду с внутренним рассеянием дифференциальных потоков на обеих границах в нем аналогично необходимо учитывать еще и входящие через обе границы дифференциальные потоки. На рисунке результаты рассеяния на границах от внутренних потоков имеют индекс in, а от внешних (для рассматриваемого слоя) дифференциальных потоков - индекс ex. Указанные индексы здесь используются лишь для пояснения физической картины рассеяния потоков на границах слоя.

На границе плоскопараллельного слоя вероятность рассеяния зависит от модуля вектора дифференциального потока и полярного угла, но не зависит от азимутального угла падения дифференциального потока на границу. Конкретное значение вектора потока будет определенным, если к модулю вектора $q$ и углу $\theta$ задать его азимутальный угол $\varphi$. Поэтому в общем виде при равновесии и небольших отличиях от него граничные условия к кинетическому уравнению в твердотельном слое со сферически симметричной зависимостью $\varepsilon(\mathbf{k})$ имеют вид:

$$
\begin{aligned}
q^{+}(a, k, \Omega) \mu= & q_{a}^{+}(k, \Omega) \mu+\int_{V_{B}^{-}} P_{a}^{-}\left(k, \Omega, k_{*}, \Omega_{*}\right) \\
& \times q^{-}\left(a, k_{*}, \Omega_{*}\right) \mu_{*} k_{*}^{2} d k_{*} d \Omega_{*}, \\
q^{-}(b, k, \Omega) \mu= & q_{b}^{+}(k, \Omega) \mu+\int_{V_{B}^{+}} P_{b}^{+}\left(k, \Omega, k_{*}, \Omega_{*}\right) \\
& \times q^{+}\left(b, k_{*}, \Omega_{*}\right) \mu_{*} k_{*}^{2} d k_{*} d \Omega_{*} .
\end{aligned}
$$

Здесь $q^{+}(a, k, \Omega)$ - модуль плотности дифференциального потока носителей заряда с волновым вектором $\mathbf{k}$, распространяющегося от границы $x=a$ в направлении $\Omega=\Omega(\theta, \varphi)$ в толщу слоя (одинакового при любом значении $\varphi), \mu=|\cos \theta|, d \Omega_{*}=\sin \theta_{*} d \theta_{*} d \varphi_{*}, q_{a}^{+}(k, \Omega)-$ модуль плотности дифференциального потока носителей, вошедшего в слой через эту границу из соседнего слоя. Он также выражается аналогичным интегралом или отсутствует (на поверхности). Вероятность рассеяния носителей любого из дифференциальных потоков $q^{-}\left(a, k_{*}, \Omega_{*}\right)$, падающих на границу изнутри слоя и дающих вклад после рассеяния в поток $q^{+}(a, k, \Omega)$ в данном слое или в выходящий в соседний слой поток $q^{-}(a, k, \Omega)$, обозначена $P_{a}^{-}\left(k, \Omega, k_{*}, \Omega_{*}\right)$ (рассеяние назад в слой при $k_{x}>0$ и выхода потока за границу $x=a$ при $\left.k_{x}<0\right)$. Все обозначения в граничном условии (2) у границы $x=b$ и пояснения к ним аналогичны по смыслу обозначениям, описанным выше для границы $x=a$.

Области интегрирования $V_{B}^{-}\left[\left[0, k_{* \max }\right] ; \Omega_{*}^{-}\right] \quad$ и $V_{B}^{+}\left[\left[0, k_{* \max }\right] ; \Omega_{*}^{+}\right] \quad$ соответствуют негативной и позитивной частям зоны Бриллюэна в симметричном виде - ячейке Вигнера-Зейца с $k_{* x}<0$ и $k_{* x}>0$. Хотя в ячейке Вигнера-Зейца $k_{* \max }$ зависит от направления $\Omega_{*}\left(\theta_{*}, \varphi_{*}\right)$, обычно верхний предел интегрирования по модулю волнового вектора $k_{*}$ заменяется на бесконечность для всех значений полярного $\theta_{*}^{+}[0, \pi / 2]$, $\theta_{*}^{-}[\pi / 2, \pi]$ и азимутального $\varphi_{*}[0,2 \pi]$ углов.

В случае проявления квантово-размерных свойств тонкого твердотельного слоя интегрирование в граничных условиях необходимо изменить в соответствии с реальным строением зоны Бриллюэна.

Подобные граничные условия к кинетическому уравнению требуются для каждого внутреннего слоя рассматриваемой твердотельной структуры. На поверхности слоя входящие дифференциальные потоки носителей отсутствуют.

Все векторы плотности дифференциальных потоков в слое и их модули выражаются через функции распределения носителей заряда: $\mathbf{q}^{+}(x, k, \Omega)=\frac{2}{(2 \pi)^{3}} \boldsymbol{v}(k, \Omega)$ $\times f(x, k, \Omega), \quad$ если $\Omega(\theta, \varphi)$ принадлежит $\Omega^{+}, \quad$ и $\mathbf{q}^{-}(x, k, \Omega)=\frac{2}{(2 \pi)^{3}} \boldsymbol{v}(k, \Omega) f(x, k, \Omega)$, если $\Omega(\theta, \varphi)$ принадлежит $\Omega^{-}$, где полусферы $\Omega^{+}$и $\Omega^{-}$соответствуют направлениям потоков носителей заряда с $k_{x}>0$ и $k_{x}<0$, а $\boldsymbol{v}(\mathbf{k})=\hbar^{-1} \nabla_{k} \varepsilon(\mathbf{k})-$ скорость носителей заряда, $\varepsilon(\mathbf{k})$ - „закон дисперсии“ - зависимость энергии носителей заряда от волнового вектора $\mathbf{k}$ (в основном у края энергетической зоны) [17]. Дифференциальные потоки в толще слоя направлены по соответствующим векторам $\mathbf{k}$, если $\varepsilon(\mathbf{k})$ является симметричной функцией (именно этот случай и рассматривается всюду здесь и далее).

Очевидно, при рассматриваемых граничных условиях имеет смысл сохранить и внутри тонкого слоя разбиение дифференциального потока в виде разности позитивно и негативно направленных дифференциальных потоков. Модуль (плотности) потока также представляется в виде разности $q(x, k, \Omega)=q^{+}(x, k, \Omega)-q^{-}(x, k, \Omega)$. Это означает, что в толще слоя кинетическое уравнение 
необходимо решать как для потока $q^{+}(x, k, \Omega)$, так и для потока $q^{-}(x, k, \Omega)$ по отдельности. Затем, используя оба граничных условия, нетрудно определить эти потоки на границах и их зависимости от координаты в толще слоя, а также координатную зависимость суммарного потока. По суммарному дифференциальному потоку можно однозначно восстановить координатную зависимость функции распределения носителей $f(x, k, \Omega)$.

Условия (1) и (2) корректно описывают физическую картину рассеяния потоков, происходящую на каждом участке реальной границы или поверхности, хотя бы локально соответствующих вышеприведенным условиям. Они физически прозрачны и имеют более общий характер, чем известные условия Фукса.

\section{4. Кинетическое уравнение для дифференциальных потоков носителей заряда в тонком плоскопараллельном слое твердотельной структуры}

Для твердотельных слоев структуры с простым строением зон стационарное кинетическое уравнение, описывающее распределение носителей заряда, хорошо известно (см., например, [17]). Интеграл столкновений $J[f]$ в общем случае нелинейно зависит от функции распределения носителей заряда $f(\mathbf{r}, \mathbf{k})$. В равновесии интеграл столкновений равен нулю.

Рассеяние носителей заряда на неоднородностях разного типа, различных примесях, фононах, электронах и других частицах существенно различается. Обычно в твердом теле присутствуют разные механизмы рассеяния носителей заряда, и учесть их точно все вместе достаточно сложно. В большинстве различных случаев рассеяния ограничиваются приближением времени релаксации, когда интеграл столкновений представляется в виде

$$
J[f(\mathbf{r}, \mathbf{k})]=-\frac{f(\mathbf{r}, \mathbf{k})-f_{0}(\mathbf{r}, \mathbf{k})}{\tau(\mathbf{k})},
$$

где $f_{0}(\mathbf{r}, \mathbf{k})$ - функция распределения носителей заряда в равновесии, $\tau(\mathbf{k})$ - время релаксации к равновесному состоянию. Иногда считается, что $\tau(\mathbf{k})^{-1}=\tau_{1}(\mathbf{k})^{-1}$ $+\tau_{2}(\mathbf{k})^{-1}+\tau_{3}(\mathbf{k})^{-1}+\ldots$, где $\tau_{i}(\mathbf{k})$ отвечают разным механизмам.

Для поправки к равновесной функции $f_{1}(\mathbf{r}, \mathbf{k})=$ $=f(\mathbf{r}, \mathbf{k})-f_{0}(\mathbf{r}, \mathbf{k})$ получается линейное уравнение

$$
\boldsymbol{v}(\mathbf{k}) \nabla_{r} f_{1}(\mathbf{r}, \mathbf{k})-\frac{e}{\hbar} \mathbf{E}_{e} \nabla_{k} f_{0}(\mathbf{r}, \mathbf{k})=-\frac{f_{1}(\mathbf{r}, \mathbf{k})}{\tau(\mathbf{k})},
$$

где $\mathbf{E}_{e}=-\nabla \varphi_{e}(\mathbf{r})$ соответствует напряженности, созданной в слое лишь внешним электрическим полем [17].

Направим ось координат $O X$ нормально макроскопическим границам плоскопараллельного твердотельного слоя, и пусть внешнее электрическое поле направлено поперек слоя (по оси $O X$ ). Вводя сферическую систему координат в $k$-пространстве (симметричной ячейке Вигнера-Зейтца), в которой ось $O X$ является полярной осью, для материалов с симметричным по $\mathbf{k}$ законом дисперсии $\varepsilon(\mathbf{k})$ уравнение (4) можно переписать в виде, удобном для твердотельных слоев с плоскопараллельным строением. В рассматриваемых условиях допустимый набор скоростей $v(k)$ в толще слоя вплоть до границ не зависит от координат. Поэтому данное уравнение для плоскопараллельного слоя можно записать в виде

$$
\begin{aligned}
\cos \theta & \frac{d}{d x} q_{e}(x, k, \Omega)+\frac{q_{e}(x, k, \Omega)}{l_{k}} \\
& =-\frac{e E_{e x}}{k_{B} T} \cos \theta q_{0}(x, k, \Omega)\left[1-f_{0}(x, k, \Omega)\right] .
\end{aligned}
$$

Здесь $l_{k}=v(k) \tau(k)-$ средняя длина пробега носителей заряда с симметричной зависимостью $\tau(k)$ и введены обычные [17] обозначения

$$
\begin{aligned}
& q_{e}(x, k, \Omega)=\frac{2}{(2 \pi)^{3}} v(k, \Omega) f_{1}(x, k, \Omega), \\
& q_{0}(x, k, \Omega)=\frac{2}{(2 \pi)^{3}} v(k, \Omega) f_{0}(x, k, \Omega)
\end{aligned}
$$

для модулей плотности неравновесных и равновесных дифференциальных потоков носителей заряда с модулем волнового вектора $k$, в малом телесном угле $\Omega$ около полярного угла $\theta$ и азимутального угла $\varphi$, а также учтено, что

$$
\begin{aligned}
& \frac{e}{\hbar} E_{e x}(x) \frac{d f_{0}(x, k, \theta)}{d \varepsilon} \frac{\partial \varepsilon(\mathbf{k})}{\partial k_{x}} \frac{1}{k_{B} T} \\
& =-\frac{e E_{e x}(x)}{k_{B} T} v(k) \cos \theta f_{0}(x, k, \theta)\left[1-f_{0}(x, k, \theta)\right] .
\end{aligned}
$$

В силу плоскопараллельного строения слоя функция распределения $f_{0}$ и поправка $f_{1}$ к ней, как и введенные дифференциальные потоки, не зависят от азимутального угла $\varphi$. Тем не менее для более детального „адресного“ обозначения дифференциальных потоков используется форма записи этих функций: $f_{0}(x, k, \Omega)$ и $f_{1}(x, k, \Omega)$, где $\Omega=\Omega(\theta, \varphi)$ определяется значениями полярного и азимутального углов в $k$-пространстве. В случае $0<\theta<\pi / 2$ описывается распространение потоков с позитивной проекцией дифференциального потока, а при $\pi / 2<\theta<\pi$ или $-\pi / 2<\theta<0-$ с негативной проекцией. Поскольку уравнение (5) линейно, общее его решение может быть представлено алгебраической суммой решений.

\section{5. Решение кинетического уравнения с интегральными граничными условиями для тонкого слоя}

Общее решение полученного кинетического уравнения (5) для дифференциальных потоков носителей заряда $q^{+}(x, k, \Omega)$ и $q^{-}(k, \Omega, x)$ в плоскопараллельном слое 
твердотельной структуры нетрудно найти, например, методом вариации постоянной. Они имеют вид:

$$
\begin{aligned}
& q^{+}(x, k, \Omega)=\left\{C_{*}^{+}(k, \Omega)-\int_{a}^{x} \beta(s) q_{0}^{+}(s, k, \Omega)\right. \\
& \left.\quad \times\left[1-f_{0}(s, k, \Omega)\right] \exp \left(\frac{s-a}{\mu l_{k}}\right) d s\right\} \exp \left(-\frac{x-a}{\mu l_{k}}\right), \\
& q^{-}(x, k, \Omega)=\left\{C_{*}^{-}(k, \Omega)+\int_{x}^{b} \beta(s) q_{0}^{-}(s, k, \Omega)\right. \\
& \left.\quad \times\left[1-f_{0}(s, k, \Omega)\right] \exp \left(-\frac{s-b}{\mu l_{k}}\right) d s\right\} \exp \left(\frac{x-b}{\mu l_{k}}\right),
\end{aligned}
$$

где $\beta(x)=\frac{e E_{e x}(x)}{k_{B} T}\left(\mathrm{~cm}^{-1}\right)$ в общем случае зависит от координаты, а независящие от $x$ величины $C_{*}^{+}(k, \Omega)$ и $C_{*}^{-}(k, \Omega)$, имеющие смысл модулей плотности дифференциальных потоков на границах $x=a$ и $x=b$, направленных в глубь слоя, определяются из граничных условий.

Линейность интегральных граничных условий (1) и (2) в каждом слое позволяет искать эти постоянные величины в виде ряда. Корректный метод выполнения этой процедуры с учетом вероятностного характера рассеяния в тонких слоях толщиной $d \approx l_{e}$ достаточно сложный, ибо рассеянно отраженные от границ дифференциальные потоки носителей могут многократно достигать противоположных границ. При этом получается, что каждый распространяющийся в слое поток взаимосвязан рассеиванием на границах с другими потоками, включая равновесные дифференциальные потоки.

Для выполнения полных расчетов в каждом случае необходимо конкретизировать зависимости вероятностей рассеяния дифференциальных потоков на границах рассматриваемого слоя $P_{a}^{-}\left(k, \Omega, k_{*}, \Omega_{*}\right)$ и $P_{b}^{+}\left(k, \Omega, k_{*}, \Omega_{*}\right)$. Тем не менее, формальное решение для постоянных величин $C_{*}^{+}(k, \Omega)$ и $C_{*}^{-}(k, \Omega)$ можно записать в виде:

$$
\begin{aligned}
C_{*}^{+} \mu= & \frac{1}{1-R_{a}^{-} e^{-\lambda} R_{b}^{+} e^{-\lambda}}\left[\left(q_{a}^{+} \mu+R_{a}^{-} e^{-\lambda} q_{b}^{-}\right)\right. \\
& \left.+\left(R_{a}^{-} e^{-\lambda} Q_{0}^{-}-R_{a}^{-} e^{-\lambda} R_{b}^{+} e^{-\lambda} Q_{0}^{+}\right)\right], \\
C_{*}^{-} \mu= & \frac{1}{1-R_{b}^{+} e^{-\lambda} R_{a}^{-} e^{-\lambda}}\left[\left(q_{b}^{-} \mu+R_{b}^{+} e^{-\lambda} q_{a}^{+}\right)\right. \\
& \left.-\left(R_{b}^{+} e^{-\lambda} Q_{0}^{+}-R_{b}^{+} e^{-\lambda} R_{a}^{-} e^{-\lambda} Q_{0}^{-}\right)\right],
\end{aligned}
$$

Здесь дробные выражения следует рассматривать как степенную сумму операторов $R_{a}^{-} e^{-\lambda} R_{b}^{+} e^{-\lambda}$ или $R_{b}^{+} e^{-\lambda} R_{a}^{-} e^{-\lambda}$, воздействующих на соответствующие плотности дифференциальных потоков в квадратных скобках. Для сокращения записи здесь введены следующие обозначения: $q_{a}^{+}=q_{a}^{+}(k, \Omega)$ и $q_{b}^{-}=q_{b}^{-}(k, \Omega)$, как и ранее, - входящие в рассматриваемый слой плотности дифференциальных потоков через границу $x=a$ и $x=b$ соответственно. Выражения для суммарных потоков

$$
\begin{aligned}
& Q_{0}^{+}=\int_{a}^{b} \beta(s) q_{0}^{+}(s, k, \Omega)\left[1-f_{0}(s, k, \Omega)\right] \exp \left(\frac{s-a}{\mu l_{k}}\right) d s, \\
& Q_{0}^{-}=\int_{a}^{b} \beta(s) q_{0}^{-}(s, k, \Omega)\left[1-f_{0}(s, k, \Omega)\right] \exp \left(\frac{s-b}{\mu l_{k}}\right) d s,
\end{aligned}
$$

где $\beta(x)$ - ранее определенный коэффициент, пропорциональный приложенному к слою внешнему электрическому полю $E_{e x}(x)$. Операторы рассеяния дифференциальных потоков определяются интегральными выражениями:

$$
\begin{aligned}
R_{b}^{+} e^{-\lambda} q_{a}^{+}= & \int_{V_{B}^{+}} P_{b}^{+}\left(k, \Omega, k_{*}, \Omega_{*}\right) \\
& \times e^{-\lambda_{*}} q_{a}^{+}\left(k_{*}, \Omega_{*}\right) \mu_{*} k_{*}^{2} d k_{*} d \Omega_{*}, \\
R_{a}^{-} e^{-\lambda} Q_{0}^{-}= & \int_{V_{B}^{-}} P_{a}^{-}\left(k, \Omega, k_{*}, \Omega_{*}\right) \\
& \times e^{-\lambda_{*}} Q_{0}^{-}\left(a, k_{*}, \Omega_{*}\right) \mu_{*} k_{*}^{2} d k_{*} d \Omega_{*},
\end{aligned}
$$

$R_{a}^{-} e^{-\lambda} R_{b}^{+} e^{-\lambda} Q_{0}^{+}$- операция рассеяния $R_{a}^{-}$„ослабленного“ потока $\left(e^{-\lambda} R_{b}^{+} e^{-\lambda} Q_{0}^{+}\right)$, аналогична предыдущему выражению и т. п., $\lambda=\frac{d}{\mu l_{k}}$, где $d=b-a-$ толщина слоя. Условные обозначения операторов выбраны в указанном виде чтобы подчеркнуть сходимость ряда.

Интегральные плотности потоков, падающие на любое сечение слоя с противоположных его сторон, определяются выражениями $Q^{-}(x)=\int_{V_{B}^{-}} q^{-}(x, k, \Omega) \mu k^{2} d k d \Omega$ и $Q^{+}(x)=\int_{V_{B}^{+}} q^{+}(x, k, \Omega) \mu k^{2} d k d \Omega$, а результирующий поток $Q(x)=Q^{+}(x)-Q^{-}(x)$. Таким образом, после нахождения постоянных величин $C_{*}^{+}(k, \Omega)$ и $C_{*}^{-}(k, \Omega)$ все плотности потоков носителей заряда в слое, и дифференциальные, и интегральные, определены полностью. Зная конкретный вид закона дисперсии $\varepsilon(\mathbf{k})$ рассматриваемого твердотельного слоя, нетрудно определить поправку $f_{1}(x, k, \Omega)$ к равновесной функции.

\section{6. Анализ решения задачи}

Полученные выражения для плотностей дифференциальных потоков $q^{+}(x, k, \Omega)$ и $q^{-}(x, k, \Omega)$, а также интегральных потоков можно упростить для толстого плоскопараллельного слоя, полагая в них $d \gg \bar{l}_{e}$. При 
этом явные выражения (7), (8) и (9), (10) позволяют оставить в дифференциальных и интегральных потоках слагаемые с однократным, двукратным и т.д. числом отражений носителей от границ слоя. Длина свободного пробега носителей заряда в данной постановке задачи зависит от модуля волнового вектора носителей заряда. Поэтому отношение $d / l_{e}(k)$ и число отражений от границы может отличаться для носителей с разными волновыми векторами. В силу этого в общем случае лучше пользоваться полными полученными выражениями для плотности дифференциальных потоков носителей.

Вопрос о корректном вычислении средней длины свободного пробега носителей заряда $\bar{l}_{e}$ требует определенности. Очевидно, для слоя металла величина $\bar{l}_{e}$ определяется в основном движением носителей заряда, энергия которых близка уровню Ферми. Для изолированного слоя диэлектрика необходимо решать аналогичные задачи для носителей заряда обоих знаков. В случае „сильной“ инжекции носителей в слой диэлектрика усреднение будет корректным, если в нем учтены лишь вошедшие в слой носители заряда с достаточной для этого энергией. Для слоя полупроводника $l_{e}(k)$ может существенно зависеть от уровня легирования, температуры, уровня инжекции носителей в рассматриваемый слой и других факторов.

Если $\bar{l}_{e}$ является статистически хорошо обусловленной величиной и вышеприведенное условие $d \gg \bar{l}_{e}$ достаточно хорошо выполняется, то постоянные $C_{*}^{+}(k, \Omega)$ и $C_{*}^{-}(k, \Omega)$, характеризующие плотности потоков на границах слоя, определяются из выражений (9) и (10), в которых дробная часть отсутствует, а также отсутствуют потоки, дважды содержащие $\exp (-\lambda)$ в квадратных скобках. В слое полупроводника роль слагаемых в квадратных скобках при функции $\exp (-\lambda)$ зависит от уровня легирования и уровня инжекции носителей.

Для слоя диэлектрика дифференциальные потоки могут иметь еще более простой вид, если собственная проводимость диэлектрика практически равна нулю, и инжектированных носителей намного больше собственных. Для диэлектрического слоя уравнение (5) следует решать с неоднородной частью, равной нулю, и учитывать лишь вошедшие в слой потоки. В этом случае, как видно из выражений (7), (8), в толстом слое диэлектрика дифференциальные потоки спадают экспоненциально, а интегральные потоки - как интегральные экспоненты второго порядка, если вошедшие в слой дифференциальные потоки не зависят от направления. Иначе эта зависимость является более сложной.

В более общем случае, если $\bar{l}_{e}$ не является статистически хорошо обусловленной величиной, то вышеприведенное условие („слой толстыйс) может выполняться достаточно хорошо лишь для некоторой части носителей заряда. При этом постоянные величины $C_{*}^{+}(k, \Omega)$ и $C_{*}^{-}(k, \Omega)$ лучше вычислять по уравнениям (9) и $(10)$ полностью.

Поскольку кинетическое уравнение (5) и граничные условия (1), (2) к нему для слоя диэлектрика аналогичны интегро-дифференциальной задаче о распространении света в плоскопараллельном слое полупроводника с рассеивающими границами [18], то и решения данных задач по форме совпадают. В случае упругого рассеяния света (при $k=k_{*}$ ) они выражаются через соответствующие резольвенты $R z_{k}^{+}\left(\Omega, \Omega_{*}\right)$ и $R z_{k}^{-}\left(\Omega, \Omega_{*}\right)$, которые зависят от параметров слоя и вероятностей рассеяния потоков на обеих границах.

В случае исследования твердотельной структуры, состоящей из нескольких слоев, необходимо учитывать, что выходящие из слоя потоки попадают в соседние слои. Следовательно, общее решение задачи должно быть согласованным по всем слоям, как в равновесии, так и при наличии приложенного к структуре внешнего напряжения. Граничные условия (1), (2) позволяют согласовать решения как в двуслойной [12], так и в многослойной структурах.

При облучении структуры потоками света (в общем случае дифференциальными) кинетическое уравнение необходимо дополнить „функцией генерации“ дифференциальных потоков носителей заряда в рассматриваемом тонком слое с учетом возможного рассеяния потоков света на границах слоя. Хотя граничные условия остаются при этом прежними, входящий в слой состав потоков носителей заряда несколько меняется за счет генерации дифференциальных потоков светом в соседних слоях. Особенно это может быть заметным для потоков, рожденных в областях у границ, толщиной менее $1-2 l_{e}$, поскольку эти потоки носителей не полностью термализованы, и часть из них в этом состоянии будет рассеиваться на границе.

Постановка задачи, ее решение и метод решения позволяют определить функциональную связь между рассеиванием дифференциальных потоков носителей на границах и распределением электрического потенциала у границы плоскопараллельного слоя в равновесии в рамках кинетической теории в приближении времени релаксации. Это удобно продемонстрировать на примере отдельного образца - толстого плоскопараллельного твердотельного слоя. В этом случае выражения (7)-(14) сильно упрощаются. В толстом слое дробной частью в выражениях (9), (10) можно пренебречь и формально положить $R_{b}^{+}=0$, а кроме этого, здесь входящие в слой дифференциальные потоки отсутствуют.

Будем считать сначала распределение электрического потенциала и поля в толще твердотельного слоя заданными, например, пусть потенциал имеет зависимость $\varphi_{v}(x)$ (если образец легирован однородно, то $\varphi_{v}$ имеет постоянное значение в толще). У поверхности имеется электрическое поле, отличное от „объемного“, которое характеризуется зависимостью $\beta_{v}(x)+\beta_{s}(x)$. Подставляя эту зависимость в уравнение $(12)$, можно найти $Q_{0}^{-}$, которое не равно нулю даже при отсутствии поля в толще слоя $\left(\beta_{v}(x)=0\right)$. Считая поле $\beta_{s}(x)$,Внешним“ (при отсутствии приложенного истинно внешнего напряжения), найдем выше изложенным способом дифференциальные потоки носителей у границы $q^{+}(x, k, \Omega)$ 
и $q^{-}(x, k, \Omega)$ с помощью уравнений $(7)-(14)$, выраженные через равновесную функцию распределения, с учетом рассеяния $q^{-}(x, k, \Omega)$ на поверхности. В выражениях (7), (8) и (11), (12) удобно положить $a=0$ и устремить $b \rightarrow \infty$, помня, что $d=b-a$. По дифференциальным потокам нетрудно определить интегральные потоки $Q^{+}(x)$ и $Q^{-}(x)$ у границы, которые содержат под интегралом $\beta_{s}(s)$ (см. уравнения (7)-(14)).

Очевидно, в равновесии интегральный поток носителей $Q(x)=Q^{+}(x)-Q^{-}(x)$, в том числе на границе слоя $x=a$, равен нулю. Приравнивая поток $Q(x)$ нулю, получим интегральное уравнение для $\beta_{s}(s)$, которое содержит вероятности рассеяния потоков на границе и равновесные характеристики и параметры функции распределения. Решая указанное уравнение, можно найти зависимость $\beta_{s}(x)$, по которой нетрудно восстановить зависимость электрического поля от координаты, а затем и потенциала у поверхности. Очевидно, функция $\beta_{s}(x)$ в толще должна стремиться к нулю, так что при этом поверхностные поле и потенциал должны совпасть с их „объемными“ значениями.

Поправки к рассматриваемым функциям можно оценить, если сложить внутреннее поле и найденное поле у поверхности, считая указанную сумму потенциалов равновесной для толщи рассматриваемого слоя, а затем повторять всю процедуру вычисления приповерхностного поля, потенциала и падающих на поверхность потоков. Ограничиваясь поправками заданной точности, можно найти указанным взаимосогласованным способом распределение электрического потенциала у поверхности и обновленную равновесную функцию распределения носителей с учетом рассеяния потоков на границе. Процедура определения потенциала и функции распределения является корректной, если зависимость вероятности рассеяния дифференциальных потоков от электрического потенциала на границе известна.

В принципе, аналогично вышеприведенное условие $Q(x)=0$ на границе двух плоскопараллельных слоев, в частности у контакта металл-полупроводник, позволяет найти функциональную связь между рассеиванием дифференциальных потоков носителей на границе и распределением электрического потенциала у границы в равновесии. Однако для корректного определения $Q^{+}(x)$ и $Q^{-}(x)$ на границе структуры в этом случае необходимо пользоваться решениями для дифференциальных потоков, согласованными по обоим слоям рассматриваемой структуры $[12,13]$. Входящие потоки на смежной границе слоев (на контакте) при этом необходимо обязательно учитывать. В силу этого данная задача получается намного сложнее рассмотренной выше.

\section{7. Результаты и выводы}

В настоящей работе обоснована актуальность постановки корректных граничных условий к кинетическому уравнению для тонкого плоскопараллельного слоя твердотельной структуры. В общем виде определены интегральные граничные условия для дифференциальных потоков носителей заряда, рассеивающихся на границах слоя в плоскопараллельной твердотельной структуре. Кинетическое уравнение в приближении времени релаксации было корректно сведено к удобной форме, согласованной с интегральными граничными условиями.

Рассмотрен метод решения полученной кинетической задачи. Из-за наличия многих признаков и различий в состоянии и строении границ слоя в общем случае корректно определить граничные условия и найти решение кинетического уравнения не представляется возможным. Для твердотельного слоя с мелким микрорельефом и плотно, практически равномерно распределенными вдоль границы мелкими неоднородностями в общем виде задача была решена.

Получены операторные выражения для плотности дифференциальных и интегральных потоков внутри слоя при заданных вероятностях рассеяния дифференциальных потоков на обеих границах, входящих плотностях дифференциальных потоков $q_{a}^{+}(k, \Omega)$ и $q_{b}^{-}(k, \Omega)$, а также равновесной функции распределения носителей заряда $f_{0}(x, k, \Omega)$. Найденные выражения являются основой для аналитического или численного решения многих задач в рамках кинетической теории при заданных зависимостях вероятностей рассеяния дифференциальных потоков на границах. Случай „зеркального отражения“ носителей границей не является исключением, но данная детализация требует отдельного рассмотрения.

Проведен небольшой общий анализ указанных выражений плотности потоков для тонких и толстых твердотельных слоев разного вида - металла, диэлектрика и полупроводника. Показана необходимость и возможность согласования общего решения задачи по всем слоям как в равновесии, так и при наличии внешнего приложенного напряжения к структуре, состоящей из нескольких твердотельных слоев.

Полученные решения позволяют корректно определить плотность потока потерь, происходящих на границе плоскопараллельной структуры, с учетом строения и состояния границы [19]. Постановка и метод решения задачи позволяют определить функциональную связь между рассеянием потоков носителей на границах и распределением электрического потенциала у границы или на контакте плоскопараллельных слоев в равновесии в рамках кинетической теории в приближении времени релаксации. Выражение плотности интегрального потока носителей заряда, найденной с помощью полученных решений рассмотренной кинетической задачи, может помочь в корректном выборе значений эффективных коэффициентов диффузии и подвижности при феноменологическом описании распространения носителей через тонкий слой.

Современные нанотехнологии позволяют менять в широких пределах микрорельеф и распределения разных мелких неоднородностей, в том числе нанокластеров, на границах и поверхностях твердотельных структур. При 
этом меняются указанные строение и состояние границы, условия и вероятности рассеяния [20] дифференциальных потоков носителей заряда как по угловому распределению, так и по модулю квазиимпульса. Следовательно, изменить условия распространения и величины потоков носителей заряда через границу и слой в зависимости от целей можно с помощью технологий, влияющих только на состояние границы. В частности, в структуре каскадного солнечного элемента согласование по фототоку через слои в некоторых пределах можно обеспечивать только изменением свойств границ слоев, а не изменением их толщины или заменой материалов твердотельных слоев. Возможно, в некоторых случаях этого можно достичь нанотекстурированием границ соответствующих слоев, практически не меняя толщину слоя.

Полученные результаты могут быть использованы при исследовании физических явлений, в частности термоэлектронной эмиссии, свойств и характеристик тонкого слоя. Они будут также полезными при изучении свойств, характеристик и параметров границы или поверхности плоскопараллельных слоев твердотельных структур различного назначения. При изучении влияния состояния границ на характеристики устройств и приборов, созданных на основе плоскопараллельных твердотельных структур, полученные результаты могут оказаться тоже весьма полезными.

\section{Список литературы}

[1] М.М. Мездрогина, М.В. Еременко, В.С. Левицкий, В.Н. Петров, Е.И. Теруков, Е.М. Кайдашев, Н.В. Лянгузов. ФТП, 49 (11), 1521 (2015).

[2] В.Г. Шенгуров, В.Ю. Чалков, С.А. Денисов, Н.А. Алябина, Д.В. Гусейнов, В.Н. Трушин, А.П. Горшков, Н.С. Волкова, М.М. Иванова, А.В. Круглов, Д.О. Филатов. ФТП, 49 (10), 1411 (2015).

[3] Т.В. Малин, А.М. Гилинский, В.Г. Мансуров, Д.Ю. Протасов, А.С. Кожухов, Е.Б. Якимов, К.С. Журавлев. ФТП, 49 (10), 1329 (2015).

[4] В.Г. Божков, Н.А. Торхов, И.В. Ивонин, В.А. Новиков. ФТП, 42 (5), 546 (2008).

[5] Н.А. Торхов, В.А. Новиков. ФТП, 43 (8), 1109 (2009).

[6] А.М. Мизеров, В.Н. Жмерик, В.К. Кайбышев, Т.А. Комиссарова, С.А. Масалов, С.В. Иванов. ФТП, 43 (8), 1096 (2009).

[7] М.А. Путято, Ю.Б. Болховитянов, А.П. Василенко, А.К. Гутаковский. ФТП, 43 (9), 1275 (2009).

[8] С.Г. Конников, А.А. Гуткин, М.В. Заморянская, Т.Б. Попова, А.А. Ситникова, А.А. Шахмин, М.А. Яговкина. ФТП, 43 (9), 1280 (2009).

[9] А.Н. Алексеев, Д.М. Красовицкий, С.И. Петров, В.П. Чалый. ФТП, 46 (11), 1460 (2012).

[10] Antonio Luque, Steven Hegedus. Handbook of photovoltaic science and engineering (Copyright (C) 2003 John Wiley \& Sons Ltd, The Atrium, Southern Gate, Chichester, West Sussex PO19 8SQ, England) p. 359.

[11] M.B. Kerimi. European Science and Technology: 7th Int. Scientific Conference. Munich, April 23-24, 2014. Materials of the Conference. V. II, p. 473.
[12] M.B. Kerimi. The Development of Science in the 21st Century: Natural and Technical Sciences. The collection of Scientific Papers, Ron Bee \& Associates, N. Y., 2015, p. $68-76$,

DOI: 10.17809/06(2015)-09. http://archiv.gpscience.org/wpcontent/uploads/2015/05/0415_ny68-76.pdf

[13] М.Б. Керими. Х Белорусско-Российский сем. „Полупроводниковые лазеры и системы“. Сб. ст., с. 165-169. 26-29 мая 2015 г. Минск, Беларусь.

[14] М.Б. Керими. Физ. основы приборостроения, 4 (3), S46 (2015).

[15] Э. Зенгуил. Физика поверхности (М., Мир, 1990).

[16] В.Л. Бонч-Бруевич, С.Г. Калашников. Физика полупроводников (М., Наука, 1977).

[17] Б.М. Аскеров. Электронные явления переноса в полупроводниках (М., Наука, 1985).

[18] М.Б. Керими. Матер. II междунар. конф. „Оптические и фотоэлектрические явления в полупроводниковых микрои наноструктурах“ (Республика Узбекистан, Фергана, 2011) c. 218-221.

[19] М.Б. Керими. Науч.-техн. журн. Ферганского Политинститута, спец. выпуск (Материалы международной конференции, 2014) с. 22-26.

[20] Г.Б. Лесовик, И.А. Садовский. УФН, 181 (10), 1041 (2011).

Редактор Г.А. Оганесян

\section{Charge carriers flow distribution into a thin layer of plane-parallel solid-state structure with scattering account on layer's boundaries}

\section{M.B. Kerimi}

\section{Centre of Technologies,}

Turkmenistan Academy of Sciences, 744000 Ashgabat, Turkmenistan

Abstract Boundaries construction of a solid-state structure thin plane-parallel layer influences onto charge carriers differential flows distribution in layer's thickness and through it. Scattering of flows on the thin layer boundaries influences onto the distribution function of charge carriers. This differential flows scattering is correctly taking into account in integrated boundary conditions to the kinetic equation. For a plane-parallel layer the kinetic equation in relaxation time approach is reduced to the convenient shape featuring distribution of flows in layer's thickness. In a general view the solution of a problem about distribution of charge carriers differential flows in structure's thin layer is gained. The analysis of the solution for different type thick and thin layer metal, dielectric, semiconductor is passed. The problems claimed by practice in which it is possible to use the gained solutions effectively are spotted. 